\title{
Artroplastia total de codo en femenino septuagenaria
}

\author{
Total elbow arthroplasty in a septuagenarian female \\ Carlos Iván Andrade Aguilar,* Edgar Turrubiates Lucero,* Luis Gerardo Domínguez Gasca* \\ Citar como: Andrade ACl, Turrubiates LE, Domínguez GLG. Artroplastia total de codo en femenino septuagenaria. \\ Acta Med Grupo Angeles. 2021; 19 (2): 285-288. https://dx.doi.org/10.35366/100457
}

\section{Resumen}

Introducción: Durante las últimas cuatro décadas, la artroplastia total de codo se ha transformado en una opción viable en aquellos incidentes con lesiones severas de esa articulación, independientemente de su etiología. Caso clínico: Femenino de 77 años, con antecedente de tres cirugías previas de codo en los dos últimos años, por inestabilidad ocasionada por rompimiento de húmero distal, sufriendo nueva caída que ocasionó fractura luxación de codo, por lo cual se realizó el citado tipo de intervención, mejorando la estabilidad y función de esa unión. Conclusiones: La artroplastia completa de codo es una alternativa en casos de articulación no reconstruible, que proporciona mejoría importante en la función, pero cuya tasa de complicaciones es alta.

Palabras clave: Artroplastia de codo, codo funcional.

\section{INTRODUCCIÓN}

La articulación del codo es esencial para movimiento de la extremidad superior, pues resulta indispensable en muchas actividades de la vida diaria. Durante las pasadas cuatro décadas, gracias a la mayor comprensión de la biomecánica de esa unión y a ciertas mejoras en el diseño de los elementos protésicos, la artroplastia total de codo (ATC) se ha transformado en una iniciativa viable en aquellos pacientes con artritis reumatoide avanzada de dicho codo, ${ }^{1}$ extendiéndose su indicación a casos de osteoartritis, artritis postraumática, fracturas humerales

\footnotetext{
* Ortopedista, Cirugía Articular. División de Cirugía del Hospital Ángeles León. León, Guanajuato, México.
}

Correspondencia:

Dr. Luis Gerardo Domínguez Gasca

Correo electrónico: luisdom88@hotmail.com

Aceptado: 15-04-2020.

www.medigraphic.com/actamedica

\section{Abstract}

Introduction: During the last four decades, total elbow arthroplasty has become a viable option for those cases with severe elbow injuries, regardless of their etiology. Clinical case: 77-year-old female, with a history of three previous surgeries of that joint in the last two years, due to instability caused by fracture of the distal humerus, suffering a new fall that caused a fractured dislocation of the elbow, for which this type of arthroplasty was performed, improving joint stability and function. Conclusions: Total arthroplasty of this joint is an option in cases of non-reconstructible elbow, that provides significant improvement in function, but which has a high rate of complications.

Keywords: Elbow arthroplasty, elbow function.

distales no reconstruibles, y otras situaciones especiales, por ejemplo artritis idiopática juvenil y resección de tumores primarios o metastásicos de codo. Con independencia de su utilidad y de la recuperación funcional que la ATC brinda, es un procedimiento que tiene una tasa elevada de complicaciones.

\section{PRESENTACIÓN DEL CASO}

Femenino de 77 años, ama de casa, con antecedentes de hipertensión arterial de siete años de evolución controlada tomando losartán, quien dos años antes sufrió rompimiento distal de húmero izquierdo, manejada con el uso de obenque a nivel de fractura de olecranon y placas medial y lateral de húmero distal, padeciendo como secuelas inestabilidad de la articulación, además de parestesias y dolor en territorio del cúbito y limitación en actividades de la vida diaria, lo cual ocasionó retiro de placa medial y liberación de su nervio cubital, pasándolo a compartimiento anterior; evolucionó con mayor inestabilidad de codo, exacerbándose la sintomatología neurológica, por lo que se realizó reconstrucción 
ligamentaria, al pasar el nervio cubital a zona anterior, sin lograr reducción de síntomas neurológicos. Se efectuó electromiografía y velocidades de conducción del mencionado nervio, reportándose mononeuropatía sensitivo-motora asimétrica de patrón axonal difuso de severa intensidad, exhibiendo cronodispersión y ondas $\mathrm{F}$ muy disminuidas.

Se le efectuó exploración del nervio cubital hallándose pinzamiento del mismo con la cabeza radial durante la flexión, volviendo a colocar el nervio en su sitio original mejorando así su sintomatología de manera paulatina; la paciente presentó una nueva caída de su altura y sufrió fractura luxación del codo (Figura 1), por lo cual se propone artroplastia total, con implante Discovery system (Zimmer-Biomet), requiriendo para su colocación: a) retiro de material de osteosíntesis previo, b) reconstrucción de la paleta humeral con rotación de tróclea pediculada con periostio, fijándola usando tornillo canulado y c) colocación de prótesis total (Figuras 2 y 3). Su evolución postquirúrgica fue satisfactoria, se le canalizó a rehabilitación, logrando la extensión completa de codo a los 30 días, flexión de $130^{\circ}$ y pronación de $70^{\circ}$ y supinación de $75^{\circ}$, con hiposensibilidad de territorio del cúbito e hipotrofia de región hipotenar y de interóseos; se manejó por cuatro meses aplicando electroestimulaciones y reeducación de músculos de la mano, recuperando así fuerza muscular de 4/5 en la escala de Daniels de los músculos flexor corto del dedo meñique, abductor del meñique y oponente del meñique, dándosele de alta con hipoestesia discreta de región cubital en mano al compararla con el lado contralateral, y permaneciendo asintomática a nueve meses de seguimiento.

\section{DISCUSIÓN}

El diseño de artroplastia total del codo (ATC) ha evolucionado en décadas recientes. ${ }^{1}$ Las indicaciones en este tipo de cirugía incluyen artritis reumatoide avanzada, osteoartritis severa, artritis postraumática, secuelas adversas de trauma y fracturas agudas no reconstruibles (semejante al caso presentado), y tiene por objetivo aliviar el dolor en la articulación mientras se mantiene un rango de movimiento funcional, ${ }^{2}$ el cual se ha definido como la flexoextensión de $30^{\circ}$ a $130^{\circ}$ y rotación de antebrazo al menos de $100^{\circ}$, correspondiendo $50^{\circ}$ a la pronación y $50^{\circ}$ a la supinación. Tales ventajas de la ATC deben sopesarse contra los riesgos de complicación y reoperación, ya que la tasa de contratiempos de la ATC es alta, pues va de 14 al 80\% según varios reportes, y tiene una media de $24 \%$, siendo comunes éstas: la fractura periprotésica, la ruptura del tríceps, quedando como más frecuente la neuropatía del cubital (frecuentemente transitoria y sensorial, como en el caso presentado); del mismo modo que el aflojamiento protésico con una tasa de $7 \%$ y de infección de $3 \%{ }^{3}$ A modo de ejemplo, en el estudio de Geurts ${ }^{4}$ y colaboradores, en un grupo de 532 pacientes con ATC, en 232 casos (44\%) se produjo cuando menos una complicación, resultando las más habituales: aflojamiento aséptico en $21 \%$ y fracturas periprotésicas en $15 \%$, ocasionando 128 reoperaciones en 116 casos (21.8\%), estas últimas implicaron revisión en $57 \%$, retiro de la prótesis en $22 \%$, cerclaje (4\%) y colocación de espaciador de remplazo en $4 \%$.

Las contraindicaciones absolutas ${ }^{5}$ para ATC son: infección activa, tejido blando local inadecuado y parálisis neuromuscular. Mientras tanto, las contraindicaciones
Figura 1:

Radiografías simples de codo izquierdo:

A) anteroposterior $y$

B) lateral, muestran fractura luxación de codo y material de osteosíntesis de lesión previa.

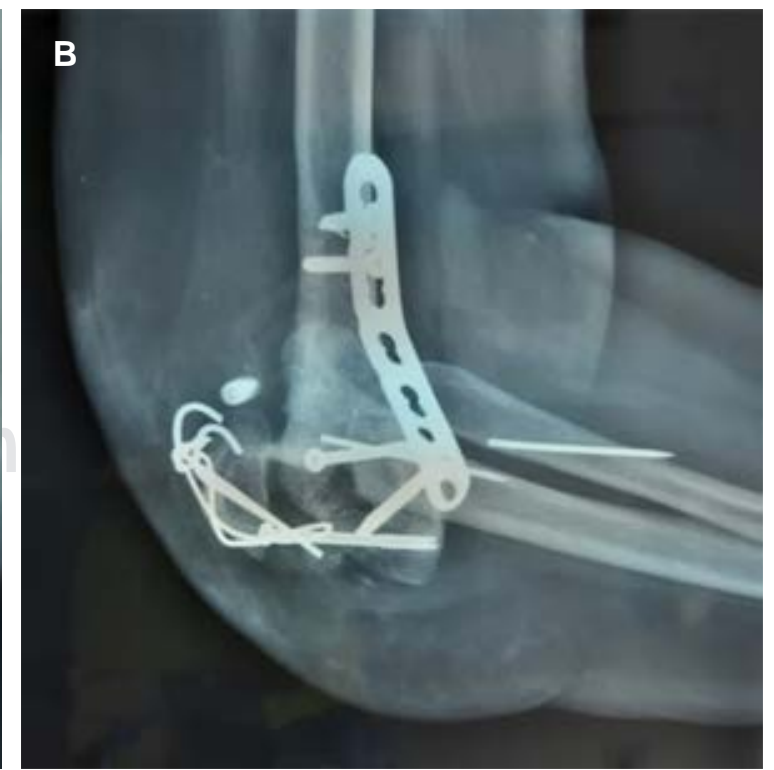



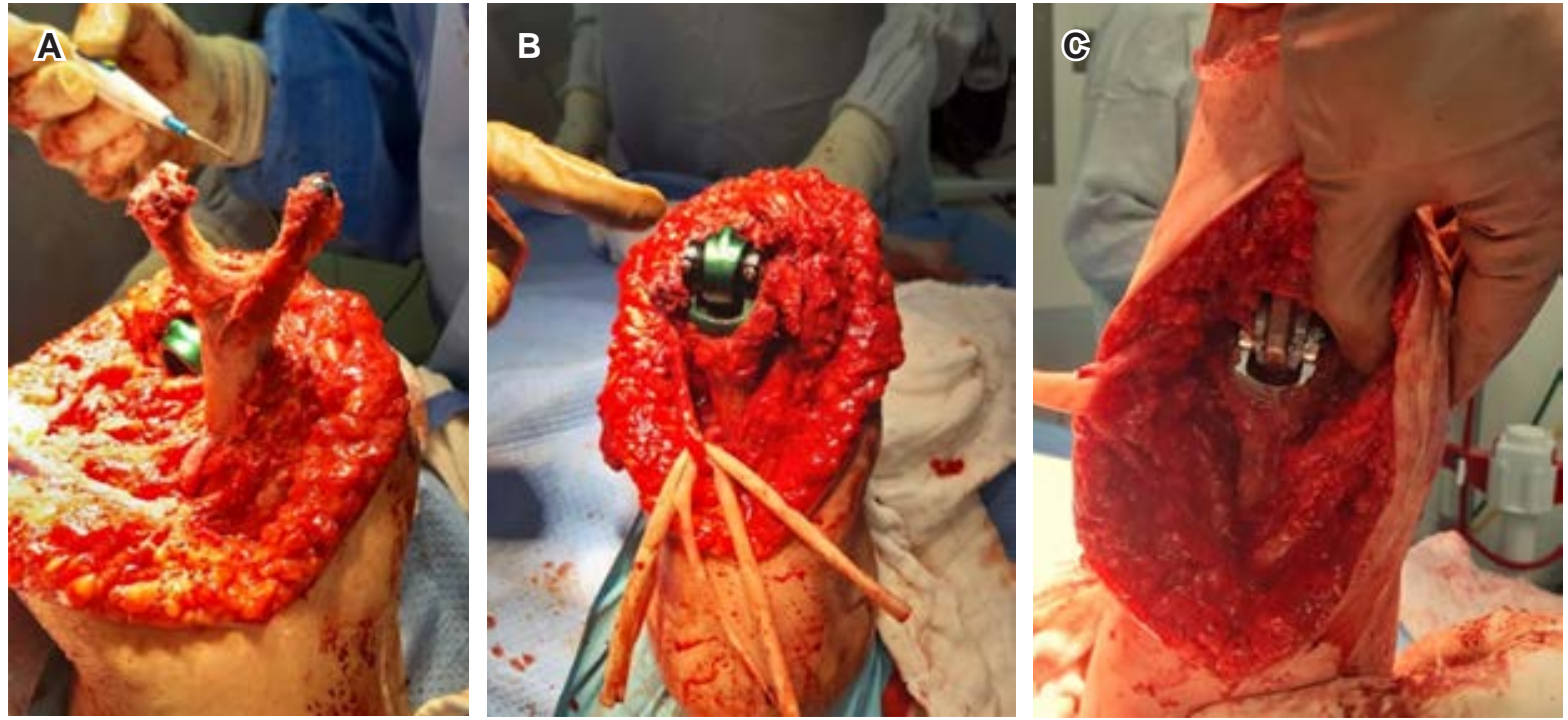

Figura 2: Fotografías clínicas de artroplastia total, se exhibe implante Discovery system (Zimmer-Biomet), requiriendo para su colocación retiro de material de osteosíntesis previo; A) reconstrucción de la paleta humeral con rotación de tróclea pediculada con periostio fijándola con tornillo canulado; B) prueba de la prótesis en flexión de codo; C) prueba de dicho elemento en extensión.
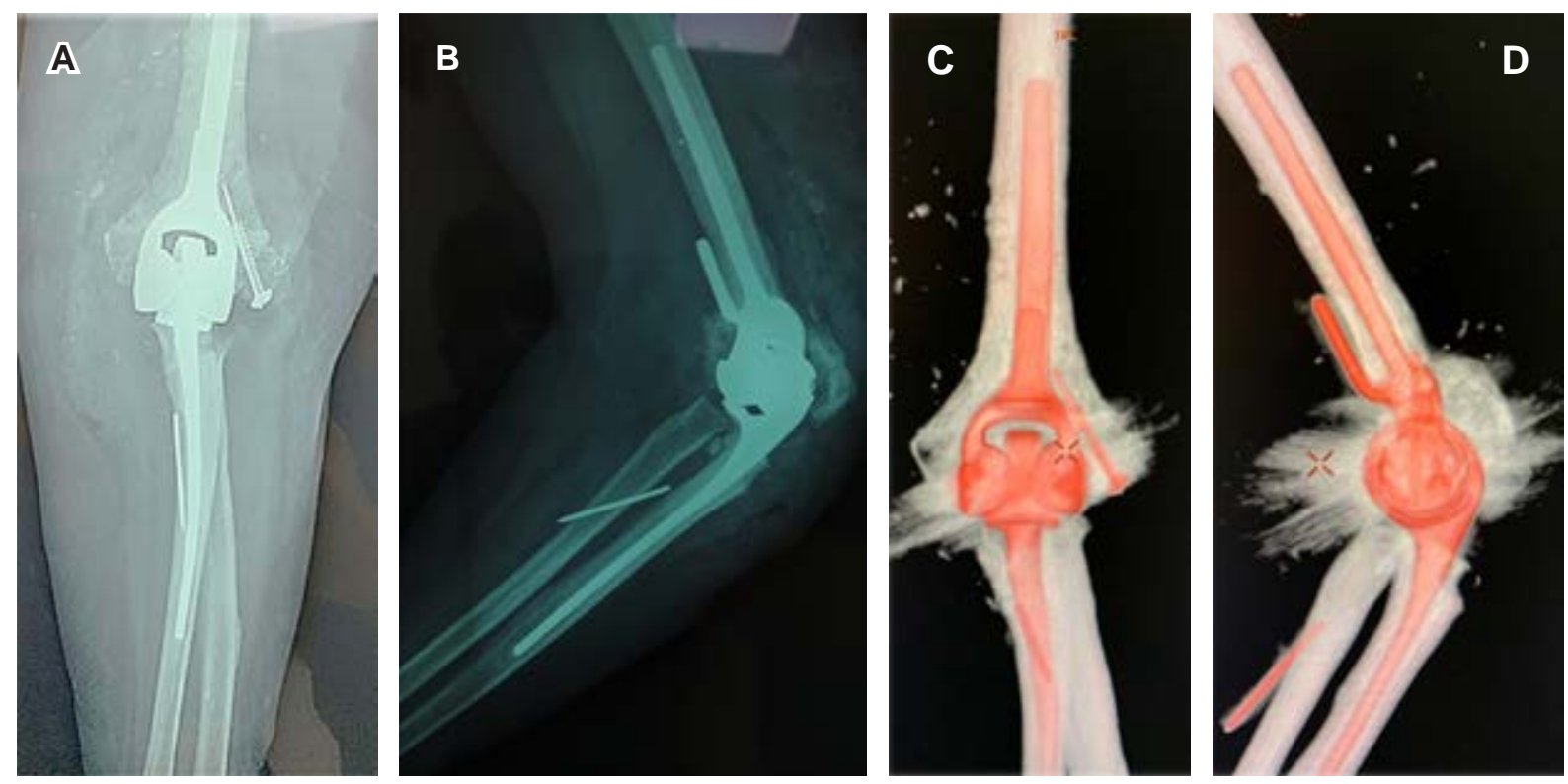

Figura 3: A y B) Imágenes radiográficas y C y D) tomográficas en vistas anteroposterior y lateral, muestran prótesis total de codo izquierdo en femenino de 77 años.

relativas son: pacientes quienes carecen de tríceps, pacientes quienes no están dispuestos o no pueden adherirse a limitaciones de actividad, trabajadores que manipulan objetos pesados y personas dependientes de dispositivos de asistencia de extremidades superiores para la ambulación.

\section{CONCLUSIONES}

La artroplastia total de codo es una opción en eventos de articulación no reconstruible, que proporciona mejoría importante en la función, pero cuya tasa de complicaciones es notoria. 


\section{REFERENCIAS}

1. Frostick SP, Elsheikh AA, Mohammed AA, Wood A. Results of cementless total elbow arthroplasty using the Discovery elbow system at a mean follow-up of 61.8 months. J Shoulder Elbow Surg. 2017; 26 (8): 1348-1354. doi: 10.1016/j.jse.2017.03.025.

2. Zhang D, Chen N. Total elbow arthroplasty. J Hand Surg Am. 2019; 44 (6): 487-495. doi: 10.1016/j.jhsa.2018.11.005.
3. Kaufmann RA, D'Auria JL, Schneppendahl J. Total elbow arthroplasty: elbow biomechanics and failure. J Hand Surg Am. 2019; 44 (8): 687-692.

4. Geurts EJ, Viveen J, van Riet RP, Kodde IF, Eygendaal D. Outcomes after revision total elbow arthroplasty: a systematic review. J Shoulder Elbow Surg. 2019; 28 (2): 381-386.

5. Park SE, Kim JY, Cho SW, Rhee SK, Kwon SY. Complications and revision rate compared by type of total elbow arthroplasty. J Shoulder Elbow Surg. 2013; 22 (8): 1121-1127. 